\title{
Nonlinear spectral compression in optical fibre: A new tool for processing degraded signals
}

\author{
Sonia Boscolo ${ }^{1}$, Frédéric Audo ${ }^{2}$, Julien Fatome ${ }^{2}$ and Christophe Finot ${ }^{2}$ \\ ${ }^{1}$ Aston Institute of Photonic Technologies, School of Engineering and Applied Science, Aston University, \\ Birmingham B4 7ET, United Kingdom \\ ${ }^{2}$ Laboratoire Interdisciplinaire Carnot de Bourgogne (ICB), UMR 6303 CNRS - Université de Bourgogne \\ Franche-Comté, 9 Avenue Alain Savary, BBP 47870, F-21078 Dijon Cedex, France \\ e-mail: christophe.finot @u-bourgogne.fr
}

\begin{abstract}
We propose two new applications of the spectral focusing by self-phase modulation that occurs in a nonlinear optical fibre. We numerically show the possibility of using nonlinear spectral compression to improve the optical signal to noise ratio and mitigate the amplitude jitter of the signal pulses. We also demonstrate experimentally that the use of spectral focusing in a combination with an external sinusoidal phase modulation achieves efficient suppression of coherent spectral background.
\end{abstract}

Keywords: Nonlinear fibre optics, spectral compression, signal processing.

\section{INTRODUCTION}

The effect of self-phase modulation (SPM) on an optical pulse propagating in a nonlinear optical fibre is ordinarily associated with broadening of the pulse spectrum [1]. However, this is not always the case with chirped pulses, where pulses with an initial negative chirp (such as that imparted by an anomalously dispersive element) can experience spectral narrowing $[2,3]$. Indeed, as a result of SPM, the propagating pulse will have the frequencies of the leading edge downshifted and the frequencies of the trailing edge upshifted. Therefore, in the case of an initially negatively chirped pulse, both the trailing and leading edges of the pulse will experience a spectral shift in the direction of the central frequency. This method of spectral compression (SC) has been implemented using various types of fibres and wavelengths [4-6]. The process can also sustain simultaneous amplification of the pulse [7], thereby providing an attractive solution to convert ultra-short pulses delivered by femtosecond oscillators into high-power, near-transform-limited picosecond pulses, and to counteract the spectrum expansion that usually occurs with the direct amplification of picosecond structures. The concept can be extended to fibre laser cavities [8].

In this paper, we propose two new applications of the nonlinear SC process. We design and numerically assess the performance of an optical power limiter combining nonlinear SC with centred optical band-pass filtering for the regeneration of intensity-modulated signals. We also experimentally demonstrate that the combination of SC with an external sinusoidal phase modulation applied to the pulses can significantly increase the extinction ratio of a signal degraded by a coherent background.

\section{OPTICAL POWER LIMITING FUNCTION}

\subsection{Impact of input signal-to-noise ratio}

Several recent theoretical and experimental studies of SC by SPM [5, 6, 9, 10] have been concerned with the reduction of the intensity level of the residual side lobes in the compressed spectrum that, in the nonlinearitydominant regime of propagation, result from the mismatch between the initial linear chirp of the pulse and the SPM-induced nonlinear chirp. However, to date, the problem of the stability of the SC process against various forms of quality degradation of the initial pulses, such as amplitude fluctuations or broadband noise, has not been yet considered. Here, we analyse the impact of amplitude jitter and degraded optical signal-to-noise ratio (OSNR) of the seed pulses on the compression process by means of extensive numerical simulations of the nonlinear Schrödinger equation. Remarkably, our results show that the spectral compression is rather stable against these pulse degradation factors. An example of this stability is illustrated in Fig. 1(a), which shows the averaged spectra of 1000 pulses after experiencing SC in a highly nonlinear fibre obtained for different input OSNR values. The pulses at the fibre input are initially 2-ps Gaussian pulses after temporal stretching by a factor of 20. It is remarkable that the OSNR of the spectrally focused pulses is nearly an order of magnitude higher than that of the seed pulses for input OSNR values above $10 \mathrm{~dB}$ [Fig. 1(b)]. The fall-off in OSNR improvement for highly degraded input pulses can be partly ascribed to the inherent sensitivity of the SPM effect driving SC to fluctuations in initial pulse duration. 

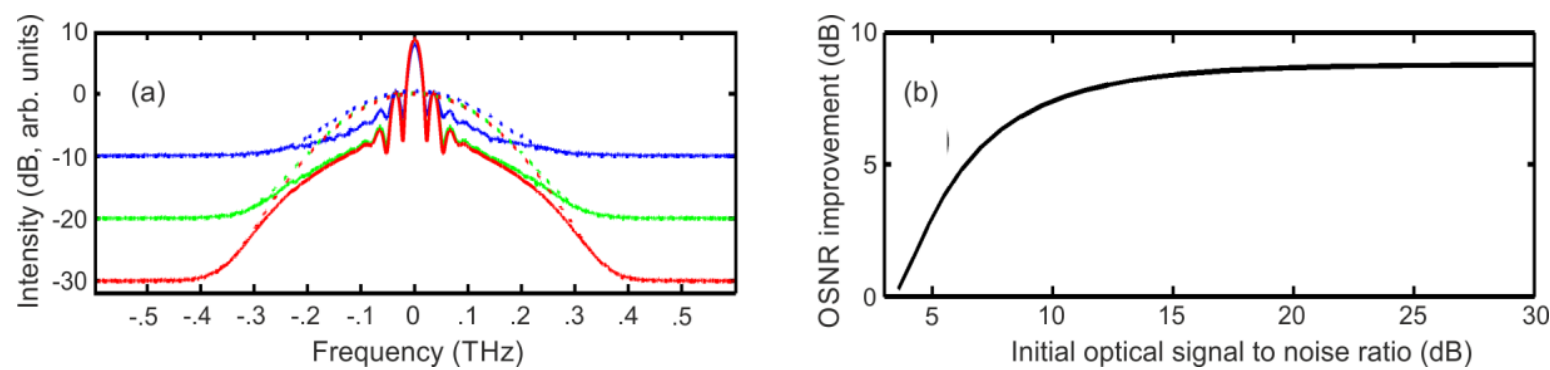

Figure 1. Impact of OSNR of the initial pulses on the SC process: (a) The averaged output spectrum of 1000 pulses at the point of optimum compression (solid) is compared with the input spectrum (dotted) for different input OSNR levels: $10 \mathrm{~dB}$ (blue), $20 \mathrm{~dB}$ (green) and $30 \mathrm{~dB}$ (red).

(b) OSNR improvement versus input OSNR.

\subsection{Potentiality of optical regeneration}

The observed stability of SC against initial pulse amplitude jitter and degraded OSNR suggested us to evaluate the capability of the process of being used in the context of optical regeneration of intensity-modulated signals. We therefore propose an optical scheme combining SC with an optical bandpass filter (OBPF) centred at the carrier frequency of the signal, which isolates the central part of the compressed spectrum, thereby converting the resulting change in central spectral brilliance into a change of the peak power of the temporal intensity profile. The computed intensity transfer function (TF) of the device [Fig. 2(a)] is shown to achieve both reduction of the amplitude fluctuations and improvement of the extinction ratio of a return-to-zero amplitudeshift keying pulse train [Fig. 2(b2), (c2)]. The statistical distribution of the peak power of the one-bit pulses highlights the reshaping of the amplitude fluctuations: at the regenerator output the distribution has no longer a Gaussian shape and is highly skewed to the left [11]. However, the amplitude noise rejection ability of the device diminishes for operation at degraded OSNR [Fig. 2(b3), (c3)]. Even though improvement of the extinction ratio is still observed, the statistical distribution of the one-bit level at the regenerator output maintains a Gaussian shape but is narrower than the input distribution. The improvement in signal quality is therefore marginal. We attribute this reduction of the device's performance to the influence of the jitter of the temporal width incurred by the input pulses because of the added amplified spontaneous emission noise. Indeed, as already demonstrated in the Mamyshev's regenerator [12], contrary to other nonlinear regenerators such as nonlinear optical loop mirrors, whose TFs only depend on the instantaneous pulse power, a variation in the input pulse duration affects the TF of our device by shifting the optimum operational power, thus impacting its beneficial effect.
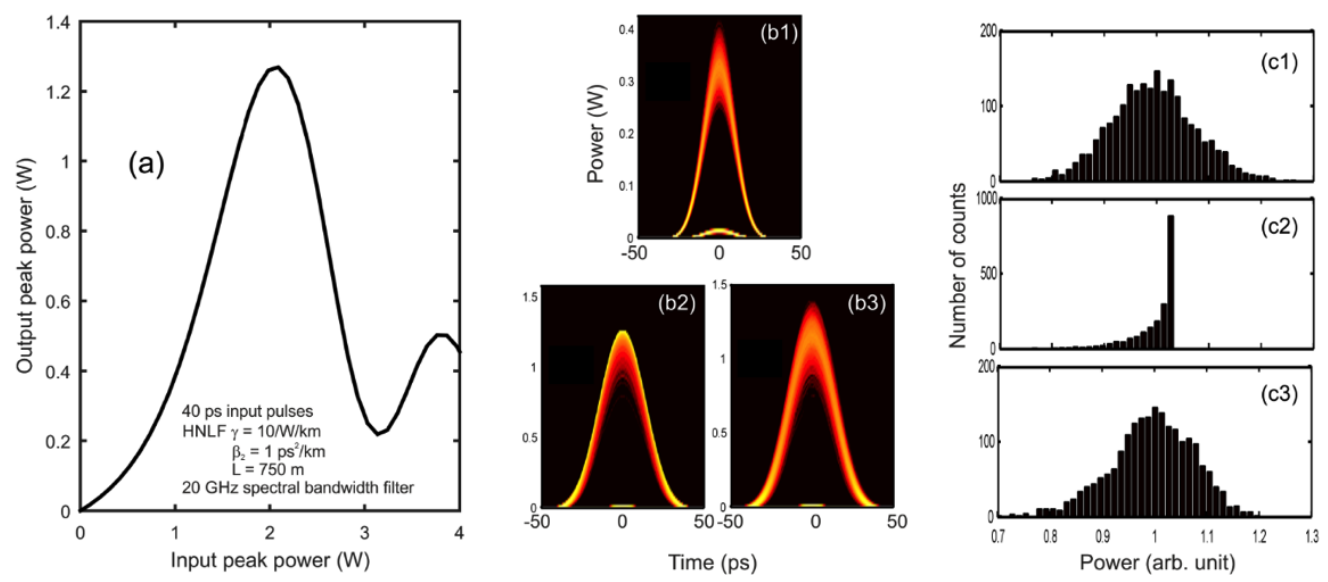

Figure 2. (a) TF of the power limiter. (b-c) Eye diagrams of a 4096 pseudorandom binary pulse train and distribution of the one-bit pulse level. Panels 1: input pulse train impaired by an rms amplitude jitter of 8\% of ideal power, filtered by a 20-GHz OBPF; panels 2: pulse train at the regenerator output; panels 3: pulse train at the regenerator output when the initial signal has an OSNR of $17 \mathrm{~dB}$. 


\section{EXTINCTION RATIO IMPROVEMENT}

\subsection{Principle of operation}

Here we describe how SC can help enhance a pulsed signal degraded by a coherent background such as, for instance, the pulses generated using a lithium niobate modulator. Because of the finite extinction ratio of the modulator (typically around $23 \mathrm{~dB}$ ), at low pulse repetition rates the energy contained in the unwanted residual background is far from negligible and may impair the practical applications of the generated pulses. The method that we propose to eliminate such spurious background is illustrated in Fig. 3 and relies on three successive stages. We first impose on the initial signal (a 120-ps Gaussian pulse with an extinction ratio of $23 \mathrm{~dB}$ in the example of Fig. 3) a sinusoidal modulation of the temporal phase with amplitude $A$ and frequency $v_{\text {mod }}$. The effect of this modulation is to create spectral replica located at frequencies $n v_{\text {mod }}$ and with amplitude $J_{n}(A)$, where $J_{n}$ is the Bessel function of the first kind of order $n$. By choosing $A=2.405$ the replica at the central frequency vanishes and all of the energy is transferred to the higher-order replicas. The second stage involves the $\mathrm{SC}$ of the modulated signal in a Kerr medium such as an optical fibre operating in the highly nonlinear regime of propagation. The effect of the SPM experienced by the propagating signal is to focus the energy back to the central frequency. It is noteworthy that the use of an initial sinusoidal temporal phase modulation is particularly well-suited to the enhancement of the performance characteristics of the SC process [10]. As the nonlinear process intrinsically discriminates the pulsed part of the signal from the coherent residual background, the energy of the background remains spread across several frequency-shifted coherent lines. Therefore, filtering the signal with an OBPF provides a simple way to remove the spurious spectral peaks and improve the extinction ratio by several tens of $\mathrm{dB}$. The resulting intensity pulse profile is slightly broadened but remains close to a Fourier-transform limited waveform.
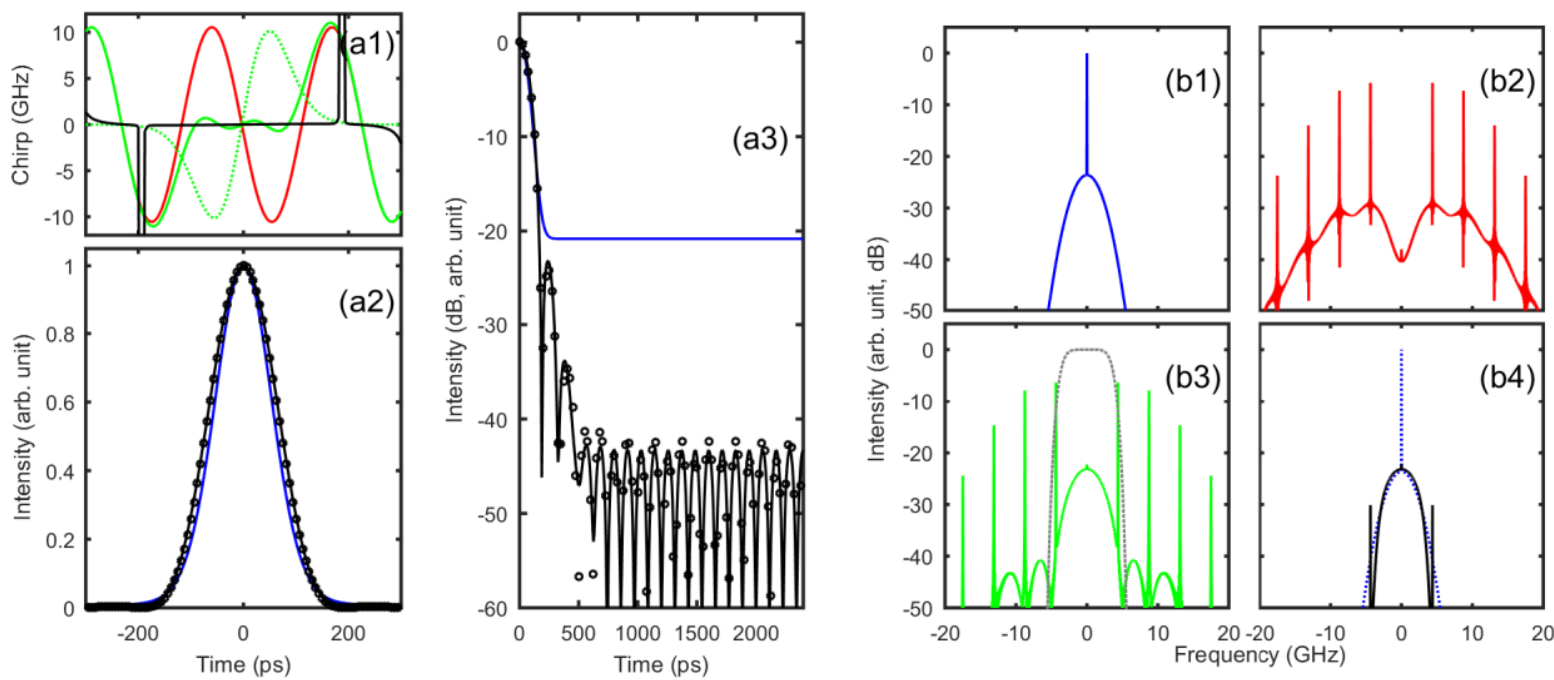

Figure 3. Operating principle of the coherent background cancellation. (a) Temporal and (b) spectral characteristics of the signal at different stages: the initial degraded signal (blue), after external phase modulation (red), after spectral focusing (green), and after band-pass filtering (black, and dotted grey in subplot b3). The circles represent the intensity profile of an equivalent transform-limited pulse.

\subsection{Proof-of-principle experiment}

We experimentally validated the proposed concept using the setup sketched in Fig. 4(a), which relies on commercially available components dedicated to optical telecommunications. Gaussian-like pulses with a fullwidth at half maximum of $120 \mathrm{ps}$ are generated at a repetition rate of $78.13 \mathrm{MHz}$. The extinction ratio of the lithium niobate modulator being used is $23 \mathrm{~dB}$, which causes a significant portion of the energy to be contained in the coherent background. The signal is first temporally phase-modulated with a sinusoidal wave of frequency 4.7 GHz and amplitude $A=2.405$. This leads to the formation of equally spaced spectral replicas, as seen in Fig. 4(c1). The spectral focusing occurs in a 500-m long section of highly non-linear fibre with a nonlinear coefficient of $10 / \mathrm{W} / \mathrm{km}$, and efficiently brings back the energy of the pulsed part of the signal to the central part of the spectrum (Fig. 4(c2), yellow curve). The energy associated the coherent background is then efficiently suppressed by a narrow-band optical filter. The resulting temporal waveform is slightly broadened compared with the initial signal but remains close to the Fourier transform limit. The estimated improvement in extinction ratio of the pulse train is more than $20 \mathrm{~dB}$. 

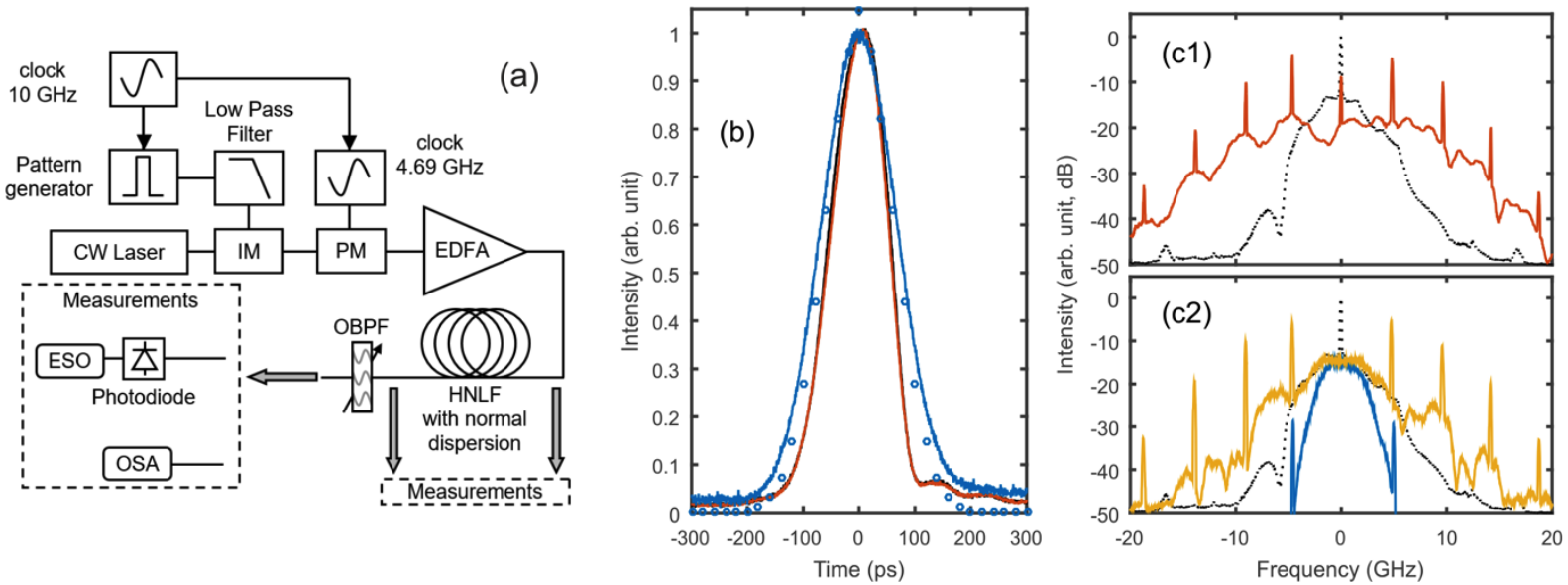

Figure 4. (a) Experimental setup. (b) Temporal intensity profile of the pulses at different stages: input profile (black), after phase modulation and spectral compression (red), and after filtering (blue). The blue open circles represent the transform-limited profile that can be derived from the spectrum after processing. (c) Spectral intensity profile of the pulses at different stages: input spectrum (black dotted line), after temporal phase modulation (red), after nonlinear propagation (yellow) and after spectral filtering (blue).

\section{CONLUSION}

We have proposed and demonstrated two new applications of the process of SC arising from nonlinear pulse propagation in an optical fibre. We have numerically assessed the performance of an optical power limiter that combines nonlinear SC with optical band-pass filtering for the regeneration of intensity-modulated signals. Further, we have presented a proof-of-concept of how SC can help improve the extinction ratio of a signal deteriorated by a coherent background.

\section{ACKNOWLEDGEMENTS}

This work was supported by the European Union within the framework of the operational Program FEDER-FSE Bourgogne 2014/2020, by the Région Bourgogne (Pari Photcom) and by the Agence Nationale de la Recherche (Labex Action ANR-11-LABX-01-01).

\section{REFERENCES}

[1] R. H. Stolen and C. Lin, "Self-phase modulation in silica optical fibers," Phys. Rev. A, vol. 17, pp. 14481453, 4/1978 1978.

[2] A. V. Zohrabian and L. K. Mouradian, "Compression of the spectrum of picosecond ultrashort pulses," Quantum Electron., vol. 25, p. 1076, 1995.

[3] M. Oberthaler and R. A. Höpfel, "Spectral narrowing of ultrashort laser pulses by self-phase modulation in optical fibers," Appl. Phys. Lett., vol. 63, pp. 1017-1019, 19931993.

[4] D. A. Sidorov-Biryukov, A. Fernandez, L. Zhu, A. Pugzlys, E. E. Serebryannikov, A. Baltuska, and A. M. Zheltikov, "Spectral narrowing of chirp-free light pulses in anomalously dispersive, highly nonlinear photonic-crystal fibers," Opt. Express, vol. 16, pp. 2502-2507, 2/2008 2008.

[5] E. R. Andresen, C. Finot, D. Oron, and H. Rigneault, "Spectral Analog of the Gouy Phase Shift," Phys. Rev. Lett., vol. 110, p. 143902, 2013.

[6] J. Fatome, B. Kibler, E. R. Andresen, H. Rigneault, and C. Finot, "All-fiber spectral compression of picosecond pulses at telecommunication wavelength enhanced by amplitude shaping," Appl. Opt., vol. 51, pp. 4547-4553, 2012.

[7] J. P. Limpert, T. Gabler, A. Liem, H. Zellmer, and A. Tünnermann, "SPM-induced spectral compression of picosecond pulses in a single-mode Yb-doped fiber amplifier," Appl. Phys. B, vol. 74, pp. 191-195, 2002 2002.

[8] S. Boscolo, S. K. Turitsyn, and C. Finot, "Amplifier similariton fiber laser with nonlinear spectral compression," Opt. Lett., vol. 37, pp. 4531-4533, 2012/11/01 2012.

[9] C. Finot and S. Boscolo, "Design rules for nonlinear spectral compression in optical fibers," J. Opt. Soc. Am. B, vol. 33, pp. 760-767, 2016.

[10] S. Boscolo, L. K. Mouradian, and C. Finot, "Enhanced nonlinear spectral compression in fibre by external sinusoidal phase modulation," J. Opt., vol. 18, p. 105504, 2016.

[11] S. Boscolo, J. Fatome, and C. Finot, "Impact of amplitude jitter and signal-to-noise ratio on the nonlinear spectral compression in optical fibres," Opt. Commun., vol. 389, pp. 197-202, 2017. 
[12] C. Finot, T. N. Nguyen, J. Fatome, T. Chartier, L. Bramerie, M. Gay, S. Pitois, and J. C. Simon, "Numerical study of an optical regenerator exploiting self-phase modulation and spectral offset filtering at $40 \mathrm{Gbit} / \mathrm{s}$, " Opt. Commun., vol. 281, pp. 2252-2264, 2008. 